\title{
A Real-Time PCR Screening Assay for Rapid Detection of Listeria Monocytogenes Outbreak Strains
}

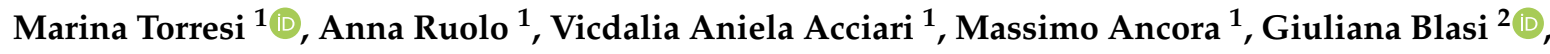 \\ Cesare Cammà ${ }^{1}$, Patrizia Centorame ${ }^{1}$, Gabriella Centorotola ${ }^{1}$, Valentina Curini ${ }^{1}{ }^{\mathbb{D}}$, \\ Fabrizia Guidi ${ }^{2} \mathbb{}$, Maurilia Marcacci ${ }^{1}$, Massimiliano Orsini ${ }^{3}$, Francesco Pomilio ${ }^{1}$ and \\ Marco Di Domenico $1, *$ (D) \\ 1 Istituto Zooprofilattico Sperimentale dell'Abruzzo e del Molise G. Caporale, via Campo Boario, \\ 64100 Teramo TE, Italy; m.torresi@izs.it (M.T.); anna.ruolo@libero.it (A.R.); v.acciari@izs.it (V.A.A.); \\ m.ancora@izs.it (M.A.); c.camma@izs.it (C.C.); p.centorame@izs.it (P.C.); g.centorotola@izs.it (G.C.); \\ v.curini@izs.it (V.C.); m.marcacci@izs.it (M.M.); f.pomilio@izs.it (F.P.) \\ 2 Istituto Zooprofilattico Sperimentale dell'Umbria e delle Marche Togo Rosati, Via Gaetano Salvemini, 1, \\ 06126 Perugia PG, Italy; g.blasi@izsum.it (G.B.); f.guidi@izsum.it (F.G.) \\ 3 Istituto Zooprofilattico Sperimentale delle Venezie, Viale dell’Università, 10, 35020 Legnaro PD, Italy; \\ morsini@izsvenezie.it \\ * Correspondence: m.didomenico@izs.it
}

Received: 6 December 2019; Accepted: 5 January 2020; Published: 8 January 2020

\begin{abstract}
From January 2015 to March 2016, an outbreak of 23 human cases of listeriosis in the Marche region and one human case in the Umbria region of Italy was caused by Listeria monocytogenes strains showing a new pulsotype never described before in Italy. A total of 37 clinical strains isolated from patients exhibiting listeriosis symptoms and 1374 strains correlated to the outbreak were received by the Italian National Reference Laboratory for L. monocytogenes (It NRL Lm) of Istituto Zooprofilattico Sperimentale dell'Abruzzo e del Molise (IZSAM) for outbreak investigation. A real-time PCR assay was purposely designed for a rapid screening of the strains related to the outbreak. PCR-positive strains were successively typed through molecular serogrouping, pulsed field gel electrophoresis (PFGE), and Next Generation Sequencing (NGS). Applying the described strategy, based on real-time PCR screening, we were able to considerably reduce time and costs during the outbreak investigation activities.
\end{abstract}

Keywords: Listeria monocytogenes; outbreak; molecular methods; real-time PCR; screening

\section{Introduction}

Listeriosis is a foodborne illness caused by Listeria monocytogenes. The most common symptoms of the mild form of the disease include diarrhoea, fever, headache, and myalgia. However, when listeriosis appears as an invasive infection, patients may develop more severe outcomes such as meningitis and/or septicemia in adults, infection of the foetus and miscarriage in pregnant women, or neonatal infection [1]. The disease is also associated with a high mortality rate, reaching $20 \%-30 \%$, and for risk-group patients even $75 \%$. The number of confirmed cases of listeriosis among the inhabitants of the EU demonstrated a growing tendency from 1439 reported in 2005 to 2549 in 2018 [2,3].

Consistent with the increasing trend at European level, an intensification in the occurrence of listeriosis was observed in Marche region, Italy, between January 2015 and May 2015. After that, a total of 24 human cases of listeriosis occurred [4].

During the outbreak a huge number of strains were sent to the Italian National Reference Laboratory for L. monocytogenes (It NRL Lm) in order to identify the source of infection, and to carry out trace back and forward activities. 
Next generation sequencing of the isolates and comparative genome analysis confirmed a unique strain responsible for the outbreak.

Since 2000, pulsed field gel electrophoresis (PFGE) has become the gold standard for L. monocytogenes subtyping and has been extensively used, throughout the world, during outbreak and trace back investigations. Although PFGE is widely accepted and used, in some cases it was found to be inadequate and obsolete [5]. Nowadays, the use of whole genome sequencing (WGS) for the characterization of pathogens has become a standard component of infectious disease surveillance and WGS-based differentiation of L. monocytogenes isolates has become a pivotal tool for listeriosis outbreak investigations in the USA [6,7]. Recent advances in sequencing technologies and analysis tools have rapidly increased the output and the analysis speed and also reduced the costs of WGS [8].

Despite this, PFGE, thanks to its internationally accredited and standardized protocol, is often joined with the Single Nucleotide Polymorphism (SNP)-based analyses [6]. In a hypothetical scenario, when outbreak occurs, thousands of samples could be processed and a huge number of L. monocytogenes strains can be isolated from human, food processing environment, and food sources leading to a possible bottleneck in both sequencing/data analysis and PFGE typing. This protocol requires up to 40 working days to accomplish strain typing. The response time of the analyses performed can make the difference between the occurrence of new clinical cases or not, and between minor or major economic loss for the food business operators and farms involved. For all these reasons the use of a screening method is a focal point for proficient outbreak management.

The aim of this paper was to describe the application of a specific real-time PCR screening assay properly designed for the rapid identification of strains potentially related to the listeriosis outbreak occurred in Central Italy between 2015 and 2016.

\section{Materials and Methods}

\subsection{Bacterial Isolates DNA Extraction}

All the strains were received as pure culture on sheep blood agar and subjected to DNA extraction. Stock cultures (Microbank ${ }^{\mathrm{TM}}$, Pro Lab Diagnostics Inc., Richmond Hill, ON, Canada) were prepared and stored at $-80^{\circ} \mathrm{C}$ when the strains were not processed immediately.

Cultures identified as L. monocytogenes by biochemical methods were grown overnight in sheep blood agar (Microbiol \& C. s.n.c., Cagliari, Italy), picked, and dissolved in $300 \mu \mathrm{L}$ of nuclease-free water (Ambion ${ }^{\mathrm{TM}}$, Thermo Fisher Scientific, Waltham, MA, USA). Then, $100 \mu \mathrm{L}$ of $20 \mathrm{mg} / \mathrm{mL}$ lysozyme was added and incubated for $2 \mathrm{~h}$ at $56^{\circ} \mathrm{C}$. Finally, $300 \mu \mathrm{L}$ of the suspension were transferred to the cartridges provided by the Maxwell 16 Cell DNA Purification Kit (Promega, Madison, WI, USA). DNA extraction was accomplished following the manufacturer's instructions. DNA was quantified using a Qubit dsDNA HS (High Sensitivity) Assay Kit (Invitrogen, Carlsbad, CA, USA) and purity was checked by a Nanodrop ND-1000 spectrophotometer (Thermo Fisher Scientific, Waltham, MA, USA).

\subsection{Serogroup}

Strain characterization was performed with a molecular serogroup-related PCR completed by the detection of flaA $[9,10]$.

Briefly, DNA was extracted as described before and reactions were carried out in a Gene Amp PCR System 9700 thermal cycler (Applied Biosystems, Foster City, CA, USA). PCR products were run in $2 \%(w / v)$ agarose gel in $1 \times$ tris/borate/EDTA (TBE) buffer (Biorad, Hercules, CA, USA) and visualized by SYBR ${ }^{\text {TM }}$ Safe DNA Gel Stain (Thermo Fisher Scientific, Waltham, MA, USA).

\subsection{PFGE}

PFGE analysis was performed, according to PulseNet protocol [11], as described previously [12]. Briefly, the bacterial suspensions were embedded in agarose, lysed, washed, and then digested with the restriction enzymes. The digested samples underwent electrophoresis in 1\% (w/v) SeaKem Gold 
agarose (Lonza Rockland, Inc., Rockland, ME, USA) in $1 \times$ TBE (Sigma-Aldrich, St. Louis, MO, USA) by using the Chef MapperXA system (Bio-Rad Laboratories, Hercules, CA, USA) at $6 \mathrm{~V} / \mathrm{cm}$, with a pulse time between 4 and $40 \mathrm{~s}$ for $19 \mathrm{~h}$.

PFGE profiles were analyzed using BioNumerics version 7.5 (Applied Maths, Sint-Martens-Latem, Belgium). The similarities between the AscI and ApaI macrorestriction profiles (MRPs) were calculated using the Dice coefficient, applying an optimization coefficient and band tolerance of $1.0 \%$ for both enzymes.

\subsection{Next Generation Sequencing}

WGS was used, in the first instance, to find common genomic regions for developing a real-time PCR screening test and then to perform outbreak inclusion/exclusions by SNPs analysis.

The DNA from L. monocytogenes strains were sequenced by the NextSeq500 Illumina platform using the Nextera XT protocol. Raw data were trimmed and assembled using Trimmomatic [13] and Spades 3.11 [14], respectively, with default parameters for the Illumina platform $2 \times 150$ chemistry. Then the genomes annotation was performed by Prokka [15] with default parameters except for the bacterial genetic code (-gcode 11). Annotation data were used to build a pan-genome matrix using roary [16] with default parameters and the gff files obtained by the annotation.

Genetic relationships among isolates and outbreak inclusion/exclusions were performed by a SNP-based approach, using the reference free tool, kSNP3 [17], and a kmer value of 21 as indicated by Morganti et al. [18]. The core SNPs matrix was used as input to draw a neighbor-joining (NJ) phylogenetic tree using Mega6 [19].

\subsection{Real-Time PCR}

Aligning whole genome sequences of 12 outbreak clinical strains by ClustalW, common genomic sequences were identified [20]. Multiple sequence alignment found 14 highly conserved regions (coding for hypothetical proteins, recombinase family protein, transcriptional regulator, DEAD-DEAH box helicase, GNAT family acetyltransferase, and N-6 DNA methylase).

Primer Express v3.0.1 software (Applied Biosystems, Foster City, CA, USA) was used to design TaqMan assays for all the 14 genome regions. The recombinase family protein gene (rec) and the transcriptional regulator (trans) assays, which showed the best score, were selected to develop a multiplex real-time PCR screening method. The assays were optimized in a duplex real-time PCR using 6-Carboxyfluorescein (FAM) and 6-Carboxy-4' 6 $^{\prime}$-Dichloro-2', $7^{\prime}$-Dimethoxyfluorescein (JOE) as fluorescent reporter dyes, respectively (Table 1).

Table 1. Primers and probes sequences for Rec and Trans real-time PCR.

\begin{tabular}{ccc}
\hline Oligonucleotide & Sequence $\mathbf{5}^{\prime} \mathbf{- 3}^{\prime}$ & Size \\
\hline Rec-fwd & AAATAATGCGGAGTTAAAAGGTGAA & \\
Rec-rev & TGGACTGCATTTGGTATGTGAGT & 74 bp \\
Rec-probe & FAM-TACGGATTGCCGTCCCCGAAAGT-BHQ1 & \\
Trans-fwd & CTCATTACGTTGATTGGCATACG & \\
Trans-rev & GGTTCGTGGTCTCCTTTTACAATAA & $79 \mathrm{bp}$ \\
Trans-probe & JOE-AACGAAGAAAAGGGAAAAACTCCCACCC-BHQ1 & \\
\hline
\end{tabular}

Oligonucleotides were synthesized by Eurofins Genomics (Ebersberg, Germany). The $20 \mu \mathrm{L}$ reaction volume contained $5 \mu \mathrm{L}$ of purified DNA $(2 \mathrm{ng} / \mu \mathrm{L}), 10 \mu \mathrm{L}$ of GoTaq Probe qPCR Master Mix $2 \times, 300 \mathrm{nM}$ for both Rec forward and reverse primers, $150 \mathrm{nM}$ for Rec probe, $600 \mathrm{nM}$ for both Trans forward and reverse primers, $200 \mathrm{nM}$ for Trans probe, and nuclease-free water up to final volume. Real-time PCR were performed on the 7900 HT Fast Real-Time PCR System (Applied Biosystems, Foster City, CA, USA) using the following thermal profile: DNA polymerase activation at $95^{\circ} \mathrm{C}$ for $20 \mathrm{~s}$ tailed by 35 cycles of denaturation at $95^{\circ} \mathrm{C}$ for $1 \mathrm{~s}$ and annealing/extension at $60^{\circ} \mathrm{C}$ for $20 \mathrm{~s}$. 
Three replicates of five ten-fold DNA serial dilutions from 20 to $0.002 \mathrm{ng} / \mu \mathrm{L}$ were amplified to create the standard curve. The efficiency $(\mathrm{E})$ was calculated according to the formula $\mathrm{E}=\left(10^{-1 / \mathrm{slope}}\right.$ 1) $\times 100[21]$.

\section{Results}

Between January 2015 and September 2016, a total number of 37 clinical strains isolated from patients exhibiting listeriosis symptoms and 1374 strains correlated to the outbreak were received by the It NRL Lm. Among them, 1397 were screened with real-time PCR, 1230 were typed with molecular serogroup-related PCR, and 490 with PFGE and WGS.

\subsection{Workflow}

Real-time PCR and serogroup determination were used as alternative screening methods to give priority for deeper typing by Next Generation Sequencing (NGS) and PFGE. Before real-time PCR, strain selection was based on serogroup determination and then PFGE. The robustness coupled with the reduced turnaround time for analysis made the real-time PCR the method of choice for screening purposes. For every sample, up to five colonies were screened and then one PCR-positive colony was sequenced by NGS and genotyped by SNPs analysis (Figure 1). All the colonies sequenced were also genotyped by PFGE.

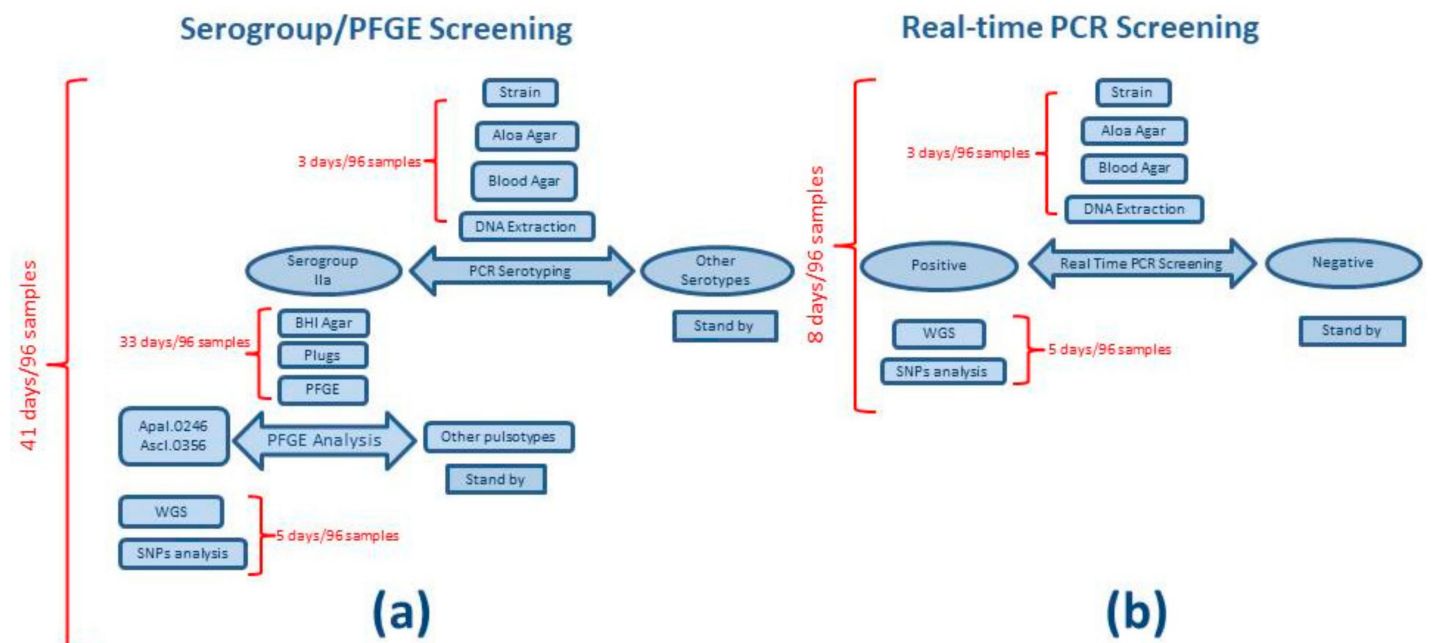

Figure 1. Analysis workflow and turnaround time to analyze 96 samples. Cases (a) and (b) show time of analysis applying serogroup and PFGE and real-time PCR as screening test to perform outbreak inclusion/exclusion, respectively.

\subsection{Serogrouping and PFGE}

Serogrouping analysis clustered 1224 isolates into four serogroups (IIa flaA+, IIc flaA-, IVb, and $\mathrm{IIb})$, one strain was associated to serogroup IIc flaAt, and five strains were identified as Listeria spp.

The main serogroup was IIa flaA+ $(47.7 \% n=587)$. Serogroup IIc flaA- was detected in $28.4 \%$ of the strains $(n=349)$, while serogroups IIb and IVb were identified in $13.3 \%(n=164)$ and $10.1 \%$ $(n=124)$, respectively.

All the strains included in the outbreak showed the same serogroup, Ila flaAt, and pulsotype ApaI.0246 AscI.0356 as defined by the European Centre for Disease Prevention and Control (ECDC).

The PFGE analysis was carried out on 490 strains with both the enzymes and yielded 97 combined pulsotypes in addition to the outbreak profile that represented the prevalent one (21.6\%) (Figure 2). 


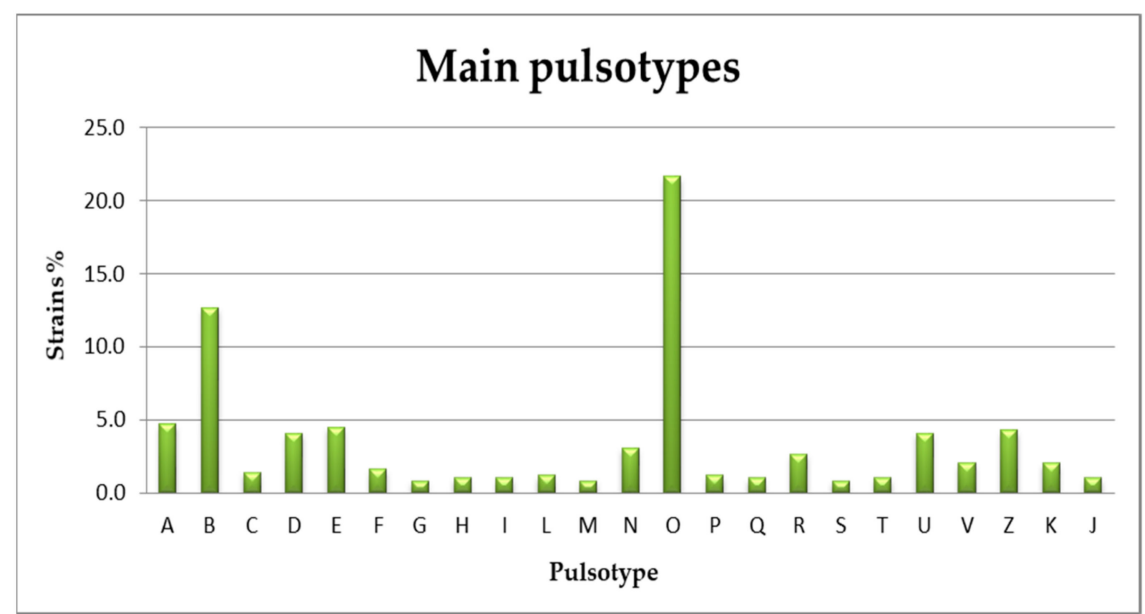

Figure 2. Main pulsotypes detected in 490 Listeria monocytogenes strains analyzed during the outbreak investigation.

\subsection{Next Generation Sequencing}

DNA of real-time PCR positive strains was sequenced by NGS and then related strains typed by PFGE. The theoretical coverage for each sample was higher than $70 \times$ with an average reads quality higher than 32.

Genetic relationships among isolates were evaluated through a SNP-based approach. The threshold for outbreak inclusion was set to 60 different SNPs. The neighbor joining phylogenetic tree showed a strong correlation between strains included in the outbreak, suggesting a high clonality of the collected strains (Figure 3). 


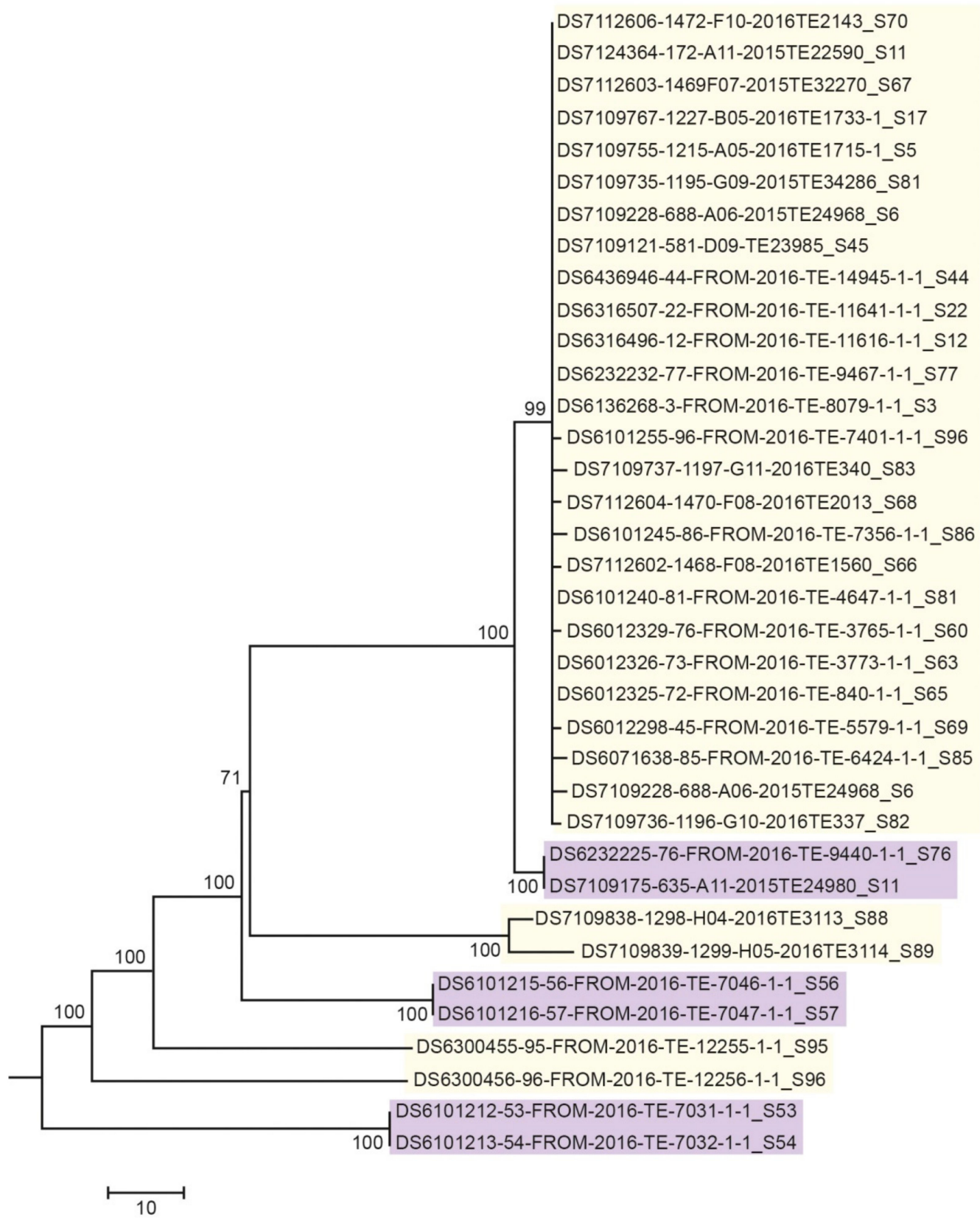

Figure 3. Phylogenetic relationships (neighbor joining (NJ)) among a selected subset of 38 strains analysed during the outbreak investigation. Strains showing pulsotype ApaI.0246 AscI.0356 are highlighted in yellow; strains positive to real-time PCR but with a different pulsotype from the outbreak strain are highlighted in purple.

\subsection{Real-Time PCR}

Analytical specificity, the ability of the assay to distinguish the DNA target from non-target DNA, was assessed on 639 strains not included in the outbreak based on SNP analysis, while 111 strains, included in the epidemic cluster, were used as positive controls.

The efficiencies of real-time PCR assays for the target Rec and Trans were $99 \%$ and $94 \%$, respectively (Table 2). All the 111 strains previously included in the outbreak by SNP analysis were correctly detected by both assays, while the DNA of the 639 strains not included in the outbreak were not amplified. The analytical specificity calculated was 100\% (lower confidence limit $99.5 \%$ and upper confidence limit $100 \%$ ). 
Table 2. Analytical performance of the duplex real-time PCR method.

\begin{tabular}{cccc}
\hline Assay & Slope & $\mathbf{R}^{2}$ & Efficiency \\
\hline Rec & -3.34 & 0.998 & $99 \%$ \\
Trans & -3.47 & 0.999 & $94 \%$ \\
\hline \multicolumn{4}{c}{ Efficiency $=\left(10^{-1 / \text { slope }}-1\right) \times 100}$.
\end{tabular}

The method was then performed on 1397 strains during the outbreak investigation. A total of 1178 $(84.3 \%)$ strains turned out to be negative, while 219 (15.7\%) were detected by both targets. All positive samples were immediately sequenced by NGS and typed by molecular serogroup-related PCR and PFGE to confirm the outbreak group identity. Twenty-three out of 219 strains were classified as PCR false positive by PFGE and SNP analysis of WGS data. Of those 23 strains, 15 strains showed different profiles respect to the outbreak pulsotype (ApaI.0246, AscI.0356). The leftover eight strains, despite having the same outbreak pulsotype, were epidemiologically unrelated and showed a number of SNPs above the cut-off (Figure 3).

\section{Discussion}

In Italy, in 2018, notification rate of invasive listeriosis was 0.29 cases per 100,000 population, lower than the average of European countries (0.47). Indeed, despite Listeria rarely exceeding the EU food safety limit tested in ready-to-eat food, many European countries reported rates higher than 0.80 (Estonia, Finland, Spain, Sweden, Denmark, Luxemburg, and Germany) [3].

Rapid identification of contaminated food and food processing industries is critical to stop the diffusion of pathogenic strains and minimize the number of cases in a foodborne outbreak. Depending on the setting (local, national, or international), one or more molecular methods needs to be carried out for typing and subtyping the involved strains.

Between January 2015 and February 2016, a total number of 63 L. monocytogenes strains isolated from food samples potentially related to the outbreak were sent to the It NRL Lm in order to identify the source of infection. In February 2016, strains isolated from a sample of hog head cheese (a typical pork-derived meat jelly-seasoned product) were identified as serotype 1/2a and showed 100\% genetic similarity with the outbreak pulsotype by PFGE [4]. After that, deep tracing back and forward investigations were carried out and 1348 strains were isolated from both contaminated food and food processing environmental samples.

Before the use of the real-time PCR method, isolates were screened by molecular serogrouping. As described in several studies [22-24], Ila is the main serogroup within strains isolated from food and food processing environments. Accordingly, in our study almost half of the typed strains were IIa flaA+, thus molecular serogrouping was not suitable for the screening purposes. Moreover, considering the number of strains received at the IT NRL Lm and the need to establish the inclusion or exclusion of food or food processing industries in the outbreak, the usual workflow was no more applicable, because the time needed to obtain results of molecular serogrouping coupled with PFGE analysis became too long.

A PCR-based method was developed to decrease the turnaround time for the screening. The real-time PCR method was able to quickly analyze up to 96 strains in $2-4 \mathrm{~h}$ and to identify all the strains potentially related to the outbreak. Positive isolates were then further investigated by PFGE and SNP analysis.

Positive results were also observed in $10.5 \%$ of strains not related to the outbreak. However, false positive outcomes are generally accepted for the inclusivity scope of a screening assay, designed to limit the number of the strains addressed for further analysis, but absolutely able to include all the strains potentially related to the outbreak.

Applying the described real-time PCR assay, we were able to drastically decrease time and costs of analysis during the outbreak. The developed strategy selected only 219 out of 587 strains, classified as 
Ila by molecular serogrouping, which were submitted to SNP analysis and PFGE with priority. As the estimated cost of PFGE per single strain is between 20.9 and 23.3 Euro, depending on the gel size [25], the gain ranged from 7691 and 8574 Euro. Moreover, the rapid response time of the analyses limited economic loss for the food business operators and farms involved. Overall, serogroup/PFGE screening was demonstrated to be less effective than the assay developed in this study.

\section{Conclusions}

WGS data were used as "starting point" to develop a PCR-based diagnostic test. We demonstrated that our novel real-time PCR assay can screen thousands of L. monocytogenes strains, significantly reducing time and costs.

Despite the fact that the method was specifically designed for L. monocytogenes strains involved in the listeriosis outbreak that occurred in Central Italy, this case report represents a proof of concept suggesting to the scientific community a new approach for any outbreak management.

WGS data were finally used as "closing point" of the workflow, the SNP-based clustering method for L. monocytogenes isolates enabled discrimination of strains indistinguishable in PFGE, but not correlated to the outbreak, and identification of the strains isolated from a hog head cheese sample produced by a facility located in Central Italy as the possible source of human infection.

Author Contributions: Authors contributed equally to this work. All authors have read and agreed to the published version of the manuscript.

Funding: This research was funded by the Italian Ministry of Health.

Acknowledgments: We thank the personnel of the local, regional and central competent authority.

Conflicts of Interest: The authors declare no conflict of interest.

\section{References}

1. Radoshevich, L.; Cossart, P. Listeria monocytogenes: Towards a complete picture of its physiology and pathogenesis. Nat. Rev. Microbiol. 2018, 16, 32-46. [CrossRef]

2. Chlebicz, A.; Śliżewska, K. Campylobacteriosis, Salmonellosis, Yersiniosis, and Listeriosis as Zoonotic Foodborne Diseases: A Review. Int. J. Environ. Res. Public Health 2018, 15, 863. [CrossRef]

3. European Food Safety Authority (EFSA) and European Centre for Disease Prevention and Control. (ECDC). The European Union summary report on trends and sources of zoonoses, zoonotic agents and food-borne outbreaks in 2018. EFSA J. 2019, 17, 5926. Available online: https:/efsa.onlinelibrary.wiley.com/doi/epdf/10. 2903/j.efsa.2019.5926 (accessed on 27 December 2019).

4. Duranti, A.; Sabbatucci, M.; Blasi, G.; Acciari, V.A.; Ancora, M.; Bella, A.; Busani, L.; Centorame, P.; Cammà, C.; Conti, F.; et al. A severe outbreak of listeriosis in central Italy with a rare pulsotype associated with processed pork products. J. Med. Microbiol. 2018, 67, 1351-1360. [CrossRef] [PubMed]

5. Pietzka, A.; Allerberger, F.; Murer, A.; Lennkh, A.; Stöger, A.; Cabal Rosel, A.; Huhulescu, S.; Maritschnik, S.; Springer, B.; Lepuschitz, S.; et al. Whole Genome Sequencing Based Surveillance of L. monocytogenes for Early Detection and Investigations of Listeriosis Outbreaks. Front. Public Health 2019, 7, 139. [CrossRef] [PubMed]

6. Datta, A.R.; Burall, L.S. Serotype to genotype: The changing landscape of listeriosis outbreak investigations. Food Microb. 2018, 75, 18-27. [CrossRef]

7. Struelens, M.J.; Brisse, S. From molecular to genomic epidemiology: Transforming surveillance and control of infectious diseases. Eurosurveillance 2013, 18, 20386. [CrossRef]

8. Quainoo, S.; Coolen, J.P.M.; van Hijum, S.A.F.T.; Huynen, M.A.; Melchers, W.J.G.; van Schaik, W.; Wertheim, H.F.L. Whole-Genome Sequencing of Bacterial Pathogens: The Future of Nosocomial Outbreak Analysis. Clin. Microbiol. Rev. 2017, 30, 1015-1063. [CrossRef]

9. Doumith, M.; Buchrieser, C.; Glaser, P.; Jacquet, C.; Martin, P. Differentiation of the Major Listeria monocytogenes Serovars by Multiplex PCR. J. Clin. Microbiol. 2004, 42, 3819-3822. [CrossRef]

10. Kérouanton, A.; Marault, M.; Petit, L.; Grout, J.; Dao, T.T.; Brisabois, A. Evaluation of a multiplex PCR assay as an alternative method for Listeria monocytogenes serotyping. J. Microbiol. Methods 2010, 80, 134-137. [CrossRef] 
11. PulseNet-International. Standard Operating Procedure for Pulsenet PFGE of Listeria monocytogenes. 2013. Available online: http://www.cdc.gov/pulsenet/PDF/listeria-pfge-protocol-508c.pdf (accessed on 21 November 2019).

12. Acciari, V.A.; Torresi, M.; Iannetti, L.; Scattolini, S.; Pomilio, F.; Decastelli, L.; Colmegna, S.; Muliari, R.; Bossù, T.; Proroga, Y.; et al. Listeria monocytogenes in smoked salmon and other smoked fish at retail in Italy: Frequency of contamination and strain characterization in products from different manufacturers. J. Food Prot. 2017, 80, 271-278. [CrossRef] [PubMed]

13. Bolger, A.M.; Lohse, M.; Usadel, B. Trimmomatic: A flexible trimmer for Illumina Sequence Data. Bioinformatics 2014, 30, 2114-2120. [CrossRef] [PubMed]

14. Bankevich, A.; Nurk, S.; Antipov, D.; Gurevich, A.A.; Dvorkin, M.; Kulikov, A.S.; Lesin, V.M.; Nikolenko, S.I.; Pham, S.; Prjibelski, A.D.; et al. SPAdes: A new genome assembly algorithm and its applications to single-cell sequencing. J. Comput. Biol. 2012, 19, 455-477. [CrossRef] [PubMed]

15. Seemann, T. Prokka: Rapid prokaryotic genome annotation. Bioinformatics 2014, 30, 2068-2069. [CrossRef]

16. Page, A.J.; Cummins, C.A.; Hunt, M.; Wong, V.K.; Reuter, S.; Holden, M.T.G.; Fookes, M.; Falush, D.L.; Keane, J.A.; Parkhill, J. Roary: Rapid large-scale prokaryote pan genome analysis. Bioinformatics 2015, 31, 3691-3693. [CrossRef]

17. Gardner, S.N.; Slezak, T.; Hall, B.G. kSNP3.0: SNP detection and phylogenetic analysis of genomes without genome alignment or reference genomes. Bioinformatics 2015, 31, 2877-2878. [CrossRef]

18. Morganti, M.; Scaltriti, E.; Cozzolino, P.; Bolzoni, L.; Casadei, G.; Pierantoni, M.; Foni, E.; Pongolini, S. Processing-Dependent and Clonal Contamination Patterns of Listeria monocytogenes in the Cured Ham Food Chain Revealed by Genetic Analysis. Appl. Environ. Microbiol. 2015, 82, 822-831. [CrossRef]

19. Tamura, K.; Stecher, G.; Peterson, D.; Filipski, A.; Kumar, S. MEGA6: Molecular Evolutionary Genetics Analysis version 6.0. Mol. Biol. Evol. 2013, 2725-2729. [CrossRef]

20. Larkin, M.A.; Blackshields, G.; Brown, N.P.; Chenna, R.; McGettigan, P.A.; McWilliam, H.; Valentin, F.; Wallace, I.M.; Wilm, A.; Lopez, R.; et al. Clustal W and Clustal X version 2.0. Bioinformatics 2007, 23, 2947-2948. [CrossRef]

21. Vaerman, J.L.; Saussoy, P.; Ingargiola, I. Evaluation of Real Time PCR data. J. Biol. Regul. Homeost. Agents 2004, 18, 212-214.

22. Lee, S.; Chen, Y.; Gorski, L.; Ward, T.J.; Osborne, J.; Kathariou, S. Listeria monocytogenes Source Distribution Analysis Indicates Regional Heterogeneity and Ecological Niche Preference among Serotype $4 \mathrm{~b}$ Clones. mBio 2018, 9. [CrossRef] [PubMed]

23. Orsi, R.H.; den Bakker, H.C.; Wiedmann, M. Listeria monocytogenes lineages: Genomics, evolution, ecology, and phenotypic characteristics. Int. J. Med. Microbiol. 2011, 301, 79-96. [CrossRef] [PubMed]

24. Pasquali, F.; Palma, F.; Guillier, L.; Lucchi, A.; De Cesare, A.; Manfreda, G. Listeria monocytogenes Sequence Types 121 and 14 Repeatedly Isolated within One Year of Sampling in a Rabbit Meat Processing Plant: Persistence and Ecophysiology. Front. Microbiol. 2018, 9, 596. [CrossRef]

25. Michelon, D.; Félix, B.; Vingadassalon, N.; Mariet, J.-F.; Larsson, J.T.; Møller-Nielsen, E.; Roussel, S. PFGE Standard Operating Procedures for Listeria monocytogenes: Harmonizing the Typing of Food and Clinical Strains in Europe. Foodborne Pathog. Dis. 2015, 12, 244-252. [CrossRef] [PubMed]

(C) 2020 by the authors. Licensee MDPI, Basel, Switzerland. This article is an open access article distributed under the terms and conditions of the Creative Commons Attribution (CC BY) license (http://creativecommons.org/licenses/by/4.0/). 